\title{
Predominance of an ST11 extended-spectrum $\beta$-lactamase-producing Klebsiella pneumoniae clone causing bacteraemia and urinary tract infections in Korea
}

Correspondence

Kwan Soo Ko

ksko@skku.edu

Jae-Hoon Song

songjh@skku.edu
Kwan Soo Ko, ${ }^{1,2}$ Ji-Young Lee, ${ }^{1}$ Jin Yang Baek, ${ }^{2}$ Ji-Yoeun Suh, ${ }^{2}$ Mi Young Lee, ${ }^{2}$ Ji Young Choi, ${ }^{1}$ Joon-Sup Yeom, ${ }^{3}$ Yeon-Sook Kim, ${ }^{4}$ Sook-In Jung, ${ }^{5}$ Sang Yop Shin, ${ }^{6}$ Sang Taek Heo, ${ }^{7}$ Ki Tae Kwon, ${ }^{8}$ Jun Seong Son, ${ }^{9}$ Shin-Woo Kim, ${ }^{10}$ Hyun-Ha Chang, ${ }^{10}$ Hyun Kyun Ki, ${ }^{11}$ Doo Ryeon Chung, ${ }^{12}$ Kyong Ran Peck ${ }^{12}$ and Jae-Hoon Song ${ }^{2,12}$

${ }^{1}$ Department of Molecular Cell Biology, Sungkyunkwan University School of Medicine, Suwon 440-746, Republic of Korea

${ }^{2}$ Asian-Pacific Research Foundation for Infectious Diseases (ARFID), Seoul 135-710, Republic of Korea

${ }^{3}$ Department of Internal Medicine, Kangbuk Samung Hospital, Sungkyunkwan University School of Medicine, Seoul 110-746, Republic of Korea

${ }^{4}$ Chungnam National University Hospital, Daejeon 301-721, Republic of Korea

${ }^{5}$ Division of Infectious Diseases, Chonnam National University Medical School, Gwangju 501-757, Republic of Korea

${ }^{6}$ Jeju National University Hospital, Jeju National University School of Medicine, Jeju 690-716, Republic of Korea

${ }^{7}$ Department of Internal Medicine, Gyeongsang National University School of Medicine and Gyeongsang Institute of Health Sciences, Jinju 660-702, Republic of Korea

${ }^{8}$ Daegu Fatima Hospital, Daegu 701-600, Republic of Korea

${ }^{9}$ East-West Neo Medical Center, Kyunghee University, Seoul 134-727, Republic of Korea

${ }^{10}$ Kyungpook National University Hospital, Daegu 700-721, Republic of Korea

${ }^{11}$ Konkuk University Hospital, Seoul 143-729, Republic of Korea

${ }^{12}$ Division of Infectious Diseases, Samsung Medical Center, Sungkyunkwan University School of Medicine, Seoul 135-710, Republic of Korea

To investigate the antimicrobial resistance, extended-spectrum $\beta$-lactamases (ESBLs) and clones of Klebsiella pneumoniae isolates causing bacteraemia or urinary tract infection (UTI) in Korea, a total of 406 K. pneumoniae isolates from patients with bacteraemia (221 isolates) and UTI (185 isolates) were collected from 10 tertiary-care Korean hospitals from July 2006 to October 2007. In vitro antimicrobial susceptibility testing was performed for all isolates and ESBL production was tested. Multilocus sequence typing (MLST) analyses were performed to characterize genotypes of ESBL-producing K. pneumoniae isolates. PFGE was performed for sequence type 11 (ST11) isolates. Forty-seven UTI isolates (25.4\%) produced ESBLs, while 30 bacteraemia isolates $(13.6 \%)$ produced ESBLs $(P=0.002)$. Among $77 \mathrm{ESBL}$-producing isolates, thirty-two (41.6\%) produced SHV-type ESBLs. bla

Abbreviations: ConNUH, Chonnam National University Hospital; CubNUH, Chungbuk National University Hospital; CunNUH, Chungnam National University Hospital; DFH, Daegu Fatima Hospital; ESBL, extended-spectrum $\beta$-lactamase; GNUH, Gyeongsang National University Hospital; JNUH, Jeju National University Hospital; KNUH, Kyungpook National University Hospital; KSH, Kangbuk Samsung Hospital; KUH, Konkuk University Hospital; MLST, multilocus sequence typing; SMC, Samsung Medical Center; ST, sequence type; UTI, urinary tract infection. 
Received 7 December 2009

Accepted 25 March 2010 in $36.4 \%$. MLST and PFGE analyses showed that ST11 was dominant in ESBL-producing K. pneumoniae isolates causing UTI (57.4\%) and in those causing bacteraemia $(70.0 \%)$ and has been prevalent in Korean hospitals. ST11 isolates harbour a combination of different ESBL genes. The ST11 clone of ESBL-producing K. pneumoniae isolates prevails in Korea, but most isolates might acquire ESBL genes independently or several different clones might be distributed in Korea.

\section{INTRODUCTION}

In Korea, the prevalence of extended-spectrum $\beta$-lactamases (ESBLs) in clinical Klebsiella pneumoniae isolates has been reported to be 17.7-30.0\% (Jeong et al., 2004; Kim et al., 2005; Ko et al., 2008a). SHV-12 is the most common ESBL in K. pneumoniae isolates from Korean hospitals followed by SHV-31, SHV-32 and TEM-52 and also CTX-M-type ESBLs, CTX-M-3, CTX-M-12, CTX-M-14 and CTX-M-15 (Bae et al., 2007; Jeong et al., 2004; Kim et al., 2005; Ko et al., 2008a; Ryoo et al., 2005). However, most of the previous studies in Korea have been performed on clinical isolates from patients irrespective of whether they cause disease.

Previously, we reported the clonal dissemination of ESBLproducing K. pneumoniae isolates in an intensive care unit in a Korean hospital based on PFGE and multilocus sequence typing (MLST) (Ko et al., 2008b). Although PFGE has been widely used for epidemiological investigation, MLST has been developed to overcome the limitations of the bandbased typing methods. Because it is highly discriminative and easy to standardize, store and exchange the information, it has been applied successfully for the epidemiological studies of many clinically important pathogens (Maiden et al., 1998). Several studies have investigated the epidemiology and characteristics of antimicrobial-resistant or virulent $K$. pneumoniae isolates using MLST (Diancourt et al., 2005; Damjanova et al., 2008; Brisse et al., 2009). In particular, Damjanova et al. (2008) showed dissemination of CTX-M-15-producing K. pneumoniae isolates belonging to limited clones in Hungary and suggested convergent population evolution in $K$. pneumoniae as in meticillinresistant Staphylococcus aureus using MLST.

In this study, we investigated the antimicrobial resistance, prevalence of ESBL, type of ESBL and genotype of $K$. pneumoniae isolates causing bacteraemia and urinary tract infections (UTIs) from 10 university hospitals in various regions of Korea from July 2006 to October 2007.

\section{METHODS}

Collection of $K$. pneumoniae isolates causing bacteraemia and UTIs. As part of a multicentre surveillance study, a total of $406 \mathrm{~K}$. pneumoniae isolates were obtained from in- and outpatients with UTIs (185 isolates) or bacteraemia (221 isolates) in 10 university hospitals in various regions of Korea: Samsung Medical Center (SMC, Seoul), Kangbuk Samsung Hospital (KSH, Seoul), Konkuk University Hospital (KUH, Seoul), Kyungpook National University Hospital (KNUH, Daegu), Daegu Fatima Hospital (DFH, Daegu), Chonnam National University Hospital (ConNUH, Gwangju), Chungnam National
University Hospital (CunNUH, Daejeon), Chungbuk National University Hospital (CubNUH, Cheongju), Gyeongsang National University Hospital (GNUH, Jinju) and Jeju National University Hospital (JNUH, Jeju). The number of isolates recovered from each hospital is described in Table 1. Only the first isolate per patient was included in the study. UTI was diagnosed according to the definitions by the Center for Disease Control and Prevention (CDC). A bacterial isolate of $\geqslant 10^{5}$ c.f.u. $\mathrm{ml}^{-1}$ in urinary culture or $\geqslant 10^{8}$ c.f.u. $\mathrm{ml}^{-1}$ in suprapubic puncture was identified as a causative pathogen of UTI. Bacteraemia was diagnosed if there were two or more of the following conditions of systemic inflammatory response syndrome: temperature of $>38{ }^{\circ} \mathrm{C}$ or $<36{ }^{\circ} \mathrm{C}$, heart rate of $>90$ beats $\mathrm{min}^{-1}$, respiratory rate of $>20$ beats $\mathrm{min}^{-1}$ or $\mathrm{PaCO}_{2}$ of $<32 \mathrm{mmHg}$, and white blood cell count of $>12000$ or $<4000$ cells $\mu^{-1},>10 \%$ bands.

Antimicrobial susceptibility testing. In vitro antimicrobial susceptibility testing was performed by a broth microdilution method according to CLSI guidelines (CLSI, 2009). Nine antimicrobial agents were tested: ampicillin, gentamicin, ciprofloxacin, ceftazidime, cefotaxime, aztreonam, imipenem, trimethoprim-sulfamethoxazole and piperacillin-tazobactam. Susceptibility interpretive criteria used were those established in CLSI standard M100-S19 (CLSI, 2009). Escherichia coli ATCC 25922, K. pneumoniae ATCC 700603 and S. aureus ATCC 29213 were used as control strains. For ESBL-positive candidates, which were screened as ceftazidime, cefotaxime or aztreonam MIC $\geqslant 2 \mathrm{mg}^{-1}$ (CLSI, 2009), production of ESBL was confirmed by a double-disc synergy test using BD BBL Sensi-Disk (Becton Dickinson), according to CLSI guidelines (CLSI, 2009). Quality control for the production of ESBL test was performed using E. coli ATCC 25922 and K. pneumoniae ATCC 700603.

Detection of bla genes. PCR and sequencing of PCR products were performed to determine the gene responsible for the ESBL activity in the ESBL producers. PCR for $b l a_{\mathrm{TEM}}, b l a_{\mathrm{SHV}}, b l a_{\mathrm{CTX}-\mathrm{M}}, b l a_{\mathrm{OXA}}$ and $b l a_{\mathrm{VEB}}$ was conducted using previously described PCR primers and conditions (Kim et al., 2005; Girlich et al., 2001). Both strands of all PCR fragments were sequenced and the types of $\beta$-lactamase genes were identified by comparing the sequences to those in the database of G. Jacoby and K. Bush (http://www.lahey.org/Studies/). ISEcp1 was

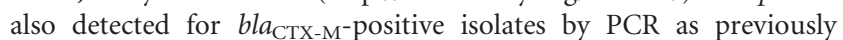
described (Damjanova et al., 2008).

MLST and PFGE. MLST was performed as described by Diancourt et al. (2005) (http://www.pasteur.fr/recherche/genopole/PF8/mlst/). Briefly stated, PCR fragments of the seven housekeeping genes rpoB, gapA, $m d h$, pgi, phoE, infB and tonB were obtained from chromosomal DNA and directly sequenced. Allelic profiles and sequence types (STs) were designated at the website (http://www.pasteur.fr/recherche/genopole/ $\mathrm{PF} 8 / \mathrm{mlst} /$ Kpneumoniae.html). New alleles and STs were submitted to the MLST website and approved. Clonal complexes were determined by including whole K. pneumoniae MLST data using the program eBURST v3 (Feil et al., 2004). For PFGE, agarose-embedded bacterial genomic

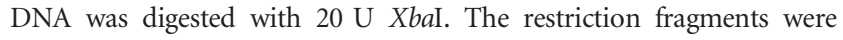
separated by electrophoresis in $0.5 \times$ TBE buffer (Chung et al., 2008). Electrophoresis was performed using CHEF Mapper XA (Bio-Rad). 
Table 1. K. pneumoniae isolates included in this study

\begin{tabular}{|c|c|c|c|c|}
\hline \multirow[t]{2}{*}{ Hospital $^{\star}$} & \multicolumn{2}{|r|}{ UTI } & \multicolumn{2}{|c|}{ Bacteraemia } \\
\hline & No. of isolates & $\begin{array}{c}\text { No. of ESBL-producing } \\
\text { isolates }\end{array}$ & No. of isolates & $\begin{array}{c}\text { No. of ESBL-producing } \\
\text { isolates }\end{array}$ \\
\hline SMC & 58 & 12 & 157 & 20 \\
\hline KSH & 19 & 5 & 2 & 1 \\
\hline KUH & 2 & $-\dagger$ & 11 & 1 \\
\hline KNUH & 4 & $-\dagger$ & 3 & $-\dagger$ \\
\hline $\mathrm{DFH}$ & 10 & 3 & NA & NA \\
\hline ConNUH & 39 & 11 & 18 & 2 \\
\hline CunNUH & 30 & 8 & 9 & 1 \\
\hline CubNUH & 5 & $-\dagger$ & 1 & 1 \\
\hline GNUH & 7 & 4 & 2 & $-\dagger$ \\
\hline JNUH & 11 & 4 & 18 & 4 \\
\hline Total & 185 & 47 & 221 & 30 \\
\hline
\end{tabular}

NA, Not available.

*SMC, Samsung Medical Center; KSH, Kangbuk Samsung Hospital; KUH, Konkuk University Hospital; KNUH, Kyungpook National University Hospital; DFH, Daegu Fatima Hospital; ConNUH, Chonnam National University Hospital; CunNUH, Chungnam National University Hospital; CubNUH, Chungbuk National University Hospital; GNUH, Gyeongsang National University Hospital; JNUH, Jeju National University Hospital.

$\dagger$ No isolates.

Statistical analysis. Fisher's exact $t$-test was used to determine the significant differences in resistance and production of ESBL using SPSS for Windows (version 11.5 software package).

\section{RESULTS AND DISCUSSION}

This study is the first nationwide surveillance and molecular characterization of invasive $K$. pneumoniae isolates from patients with bacteraemia or UTI in Korea.

\section{Antimicrobial resistances and ESBL prevalence}

The first finding from this study was that $K$. pneumoniae isolates from bloodstream infections showed lower antimicrobial resistance rates than isolates from urine cultures, although this was not significant for ampicillin, imipenem and trimethoprim-sulfamethoxazole. While resistance rate was the highest for ampicillin (93.1\%) and the lowest for imipenem (0.3\%) among 406 invasive $K$. pneumoniae isolates, the resistance rates to the other antimicrobial agents ranged from $18.2 \%$ (cefotaxime) to $24.9 \%$ (ceftazidime) (Table 2). Although all ESBLproducing members of the Enterobacteriaceae are considered resistant to third-generation cephalosporins, three and five $K$. pneumoniae isolates had MICs in the susceptibility range of cefotaxime $\left(8 \mathrm{mg} \mathrm{l}^{-1}\right)$ and ceftazidime $\left(4-8 \mathrm{mg} \mathrm{l}^{-1}\right)$, respectively. $K$. pneumoniae isolates from UTIs were more frequently ESBL producers than those from bacteraemia $(25.4 \%$ vs $13.6 \% ; P=0.002)$. Among 47 ESBL-producing isolates from patients with UTIs, 12 isolates were from SMC, 11 from ConNUH, eight from CunNUH, five from $\mathrm{KSH}$, four each from GNUH and JNUH, and three from DFH. Among 30 ESBL-producing isolates from patients with bacteraemia, 20 isolates were from SMC, four from JNUH, two from ConNUH, and one each from KSH, KNUH, CunNUH and CubNUH (Table 1). Resistance rates of clinical $K$. pneumoniae isolates in a previous (June-August 2005) investigation were similar to those in the present study (Ko et al., 2008a).

For gentamicin, ciprofloxacin, ceftazidime, cefotaxime, aztreonam and piperacillin-tazobactam, antimicrobial resistance rates were significantly lower in isolates from blood than in those from urine (Table 2). The lower resistance rates in isolates from blood may be associated with lower ESBL production rates in them. Lower resistance rates and lower prevalence rate of ESBL in $K$. pneumoniae isolates from bloodstream infections have been documented in previous studies (Jones et al., 2004; Kolar et al., 2006). However, the reason for lower resistance rates in K. pneumoniae isolates from blood is unclear.

As shown in Table 2, one K. pneumoniae isolate from blood showed imipenem resistance. This isolate was resistant to all antimicrobial agents tested in this study except trimethoprim-sulfamethoxazole. However, it did not produce ESBL. A modified Hodge test was performed for this imipenem-resistant isolate to screen for carbapenemase activity (Lee et al., 2001). As a result, the imipenem-resistant isolate was positive for carbapenemase activity. 
Table 2. Prevalence of antimicrobial resistance and ESBLs in $K$. pneumoniae UTI and bacteraemia isolates

Results are given as the number of resistant isolates (\%).

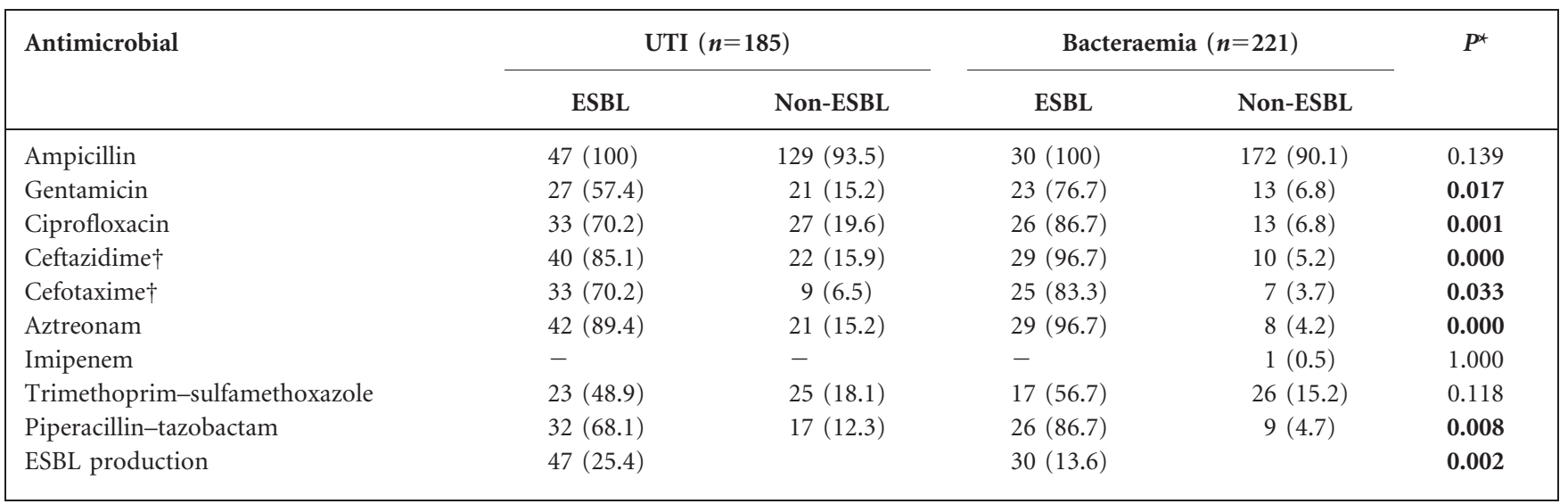

${ }^{\star} P$-value compared between total isolates from UTI and bacteraemia. Bold indicates significance.

$\dagger$ Although several ESBL-producing K. pneumoniae isolates have MICs in the susceptible range for ceftazidime and cefotaxime, they are considered resistant to them.

\section{Types of bla genes}

Among 77 ESBL-producing K. pneumoniae isolates, nine different types of ESBL genes were identified: one TEM, four SHVs and four CTX-Ms (Table 3). The most prevalent ESBL gene was SHV-12 (17 isolates, $22.1 \%$ ), followed by CTX-M-15 (14 isolates, 18.2\%), SHV-31 (13 isolates, $16.9 \%$ ) and CTX-M-14 (9 isolates, $11.7 \%$ ). While CTXM-15 was identified in $10.6 \%$ of isolates from UTIs, it was the most prevalent type in bacteraemia isolates $(30.0 \%)$. Although TEM-135, SHV-22 and CTX-M-27 were not

Table 3. Prevalence of bla genes

\begin{tabular}{|c|c|c|c|}
\hline bla gene & $\begin{array}{c}\text { Total }(n=77) \\
(\%)\end{array}$ & $\begin{array}{c}\text { UTI }(n=47) \\
(\%)\end{array}$ & $\begin{array}{l}\text { Bacteraemia } \\
(n=30)(\%)\end{array}$ \\
\hline \multicolumn{4}{|l|}{$b l a_{\mathrm{TEM}}$} \\
\hline$b l a_{\text {TEM-1 }}$ & $23(29.9)$ & $13(27.7)$ & $10(33.3)$ \\
\hline$b l a_{\mathrm{TEM}-135}$ & $1(1.3)$ & $1(2.1)$ & - \\
\hline \multicolumn{4}{|l|}{$b l a_{\mathrm{SHV}}$} \\
\hline$b l a_{\mathrm{SHV}-1}$ & $11(14.3)$ & $4(8.5)$ & $7(23.3)$ \\
\hline$b l a_{\mathrm{SHV}-11}$ & $32(41.6)$ & $22(46.8)$ & $10(33.3)$ \\
\hline$b l a_{\mathrm{SHV}-12}$ & $17(22.1)$ & $10(21.3)$ & $7(23.3)$ \\
\hline$b l a_{\mathrm{SHV}-26}$ & $2(2.6)$ & $2(4.3)$ & - \\
\hline$b l a_{\mathrm{SHV}-31}$ & $13(16.9)$ & $8(17.0)$ & $5(16.7)$ \\
\hline$b l a_{\mathrm{SHV}-93}$ variant $^{*}$ & $2(2.6)$ & $1(2.1)$ & $1(3.3)$ \\
\hline \multicolumn{4}{|l|}{ bla $_{\mathrm{CTX}-\mathrm{M}}$} \\
\hline$b l a_{\mathrm{CTX}-\mathrm{M}-14}$ & $9(11.7)$ & $7(14.9)$ & $2(6.7)$ \\
\hline$b l a_{\mathrm{CTX}-\mathrm{M}-15}$ & $14(18.2)$ & $5(10.6)$ & $9(30.0)$ \\
\hline$b l a_{\mathrm{CTX}-\mathrm{M}-22}$ & $4(5.2)$ & $3(6.4)$ & $1(3.3)$ \\
\hline$b l a_{\mathrm{CTX}-\mathrm{M}-27}$ & $1(1.3)$ & $1(2.1)$ & - \\
\hline None of the above & $19(24.7)$ & $13(27.7)$ & $6(20.0)$ \\
\hline
\end{tabular}

${ }^{*}$ Differs from SHV-93 by A79T. found in bacteraemia isolates, there were no significant differences in distribution of ESBL genes between UTI and bacteraemia isolates. Although most ESBL-producing isolates contained one ESBL gene, SHV-12 and CTX-M14, SHV-26 and CTX-M-15, SHV-31 and CTX-M-15, and SHV-31 and CTX-M-22 were identified simultaneously in each isolate. Nineteen isolates possessed SHV-11, but no ESBL genes were found in them. Their ESBL activities may be due to different ESBLs or yet-to-be identified ESBL genes. Alternatively, it is possible that we could not detect the bla genes due to nucleotide changes at the primer sites.

It has been suggested that ISEcp1 may play an important role in the mobility of the $b a_{\mathrm{CTX}-\mathrm{M}}$ genes as a promoter (Kolar et al., 2006). ISEcp1 was detected in all but one

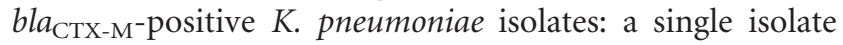
with CTX-M-27 was not positive for PCR assay of ISEcp1. However, when association of ISEcp1 with CTX-M was screened by a combination of an ISEcp1 forward primer and CTX-M reverse primer (Poirel et al., 2005), additional

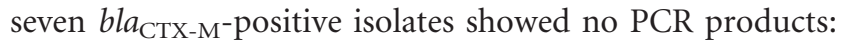
four CTX-M-14-producing isolates, two CTX-M-15-producing isolates and one CTX-M-22-producing isolate. This may imply that diverse $b l_{\text {CTX-M }}$ genes have been incorporated in K. pneumoniae isolates from Korea.

\section{Genotypes}

Genotypes of 77 ESBL-producing K. pneumoniae isolates were determined using MLST. As a result, a total of 22 different STs were identified in this study (Table 4). Of these, 19 STs belonged to 14 clonal complexes, but the other 3 STs were designated singletons. The most prevalent was ST11 (allelic profile 3-3-1-1-1-1-4). ST11 accounted for $57.4 \%$ and $70.0 \%$ of isolates from UTIs 
Table 4. Distribution of sequence types in ESBL-producing K. pneumoniae isolates

\begin{tabular}{|c|c|c|c|c|c|}
\hline \multirow[t]{2}{*}{ Clonal complex } & \multirow[t]{2}{*}{ Sequence type } & \multirow[t]{2}{*}{ Allelic profile ${ }^{\star}$} & \multicolumn{2}{|c|}{ No. of isolates } & \multirow[t]{2}{*}{ ESBL gene (no. of isolates) } \\
\hline & & & UTI $(n=47)$ & Bacteraemia $(n=30)$ & \\
\hline \multirow[t]{11}{*}{ CC11 } & ST11 & $3-3-1-1-1-1-4$ & $27(57.4 \%)$ & $21(70.0 \%)$ & SHV-12 (12) \\
\hline & & & & & SHV-12+CTX-M-14 (1) \\
\hline & & & & & SHV-31 (9) \\
\hline & & & & & SHV-31 + CTX-M-15 (1) \\
\hline & & & & & SHV-31 + CTX-M-22 (1) \\
\hline & & & & & CTX-M-15 (4) \\
\hline & & & & & CTX-M-14 (1) \\
\hline & & & & & CTX-M-22 (3) \\
\hline & & & & & Unknown (16) \\
\hline & ST258 & $3-3-1-1-1-1-79$ & 1 & 1 & CTX-M-14 (2) \\
\hline & ST270 & $3-3-1-1-1-1-23$ & $-\dagger$ & 1 & SHV-31 (1) \\
\hline \multirow[t]{2}{*}{ CC14 } & ST15 & $1-1-1-1-1-1-1$ & - & 1 & CTX-M-15 (1) \\
\hline & ST265 & $31-1-1-1-1-1$ & 1 & & SHV-12 (1) \\
\hline \multirow[t]{3}{*}{ CC37 } & ST37 & $2-9-2-1-13-1-16$ & 2 & - & TEM-35 + SHV-12 (1) \\
\hline & & & & & CTX-M-15 (1) \\
\hline & ST271 & $2-9-2-1-13-1-81$ & - & 1 & CTX-M-15 (1) \\
\hline \multirow[t]{2}{*}{$\mathrm{CC} 17$} & ST17 & $2-1-1-1-4-4-4$ & - & 1 & CTX-M-15 (1) \\
\hline & ST261 & $2-1-1-1-4-27-12$ & 2 & - & CTX-M-14 (2) \\
\hline CC292 & ST268 & $2-1-2-1-7-1-81$ & 1 & - & Unknown (1) \\
\hline CC65 & ST262 & $2-1-1-1-10-4-43$ & 1 & 1 & SHV-93 var. (2) \\
\hline \multirow[t]{2}{*}{ CC122 } & ST34 & $2-3-6-1-9-7-4$ & 2 & - & SHV-26+CTX-M-15 (1) \\
\hline & & & & & SHV-26 (1) \\
\hline CC219 & ST107 & $2-1-2-17-27-1-39$ & 1 & - & CTX-M-14 (1) \\
\hline CC163 & ST218 & $2-3-1-1-9-4-12$ & 2 & 1 & CTX-M-15 (3) \\
\hline CC133 & ST269 & $12-1-1-2-5-1-18$ & - & 1 & SHV-12 (1) \\
\hline CC285 & ST264 & $16-24-21-27-58-22-80$ & 1 & - & SHV-12 (1) \\
\hline CC39-224‡ & ST39 & $2-1-2-4-9-1-14$ & 1 & - & CTX-M-14 (1) \\
\hline CC43-101‡ & ST101 & $2-6-1-5-4-1-6$ & 1 & - & SHV-31 (1) \\
\hline CC190-263-286末 & ST263 & $2-3-1-20-7-1-16$ & 1 & - & CTX-M-14 (1) \\
\hline \multirow[t]{3}{*}{ Singleton } & ST48 & $2-5-2-2-7-1-10$ & - & 1 & CTX-M-15 (1) \\
\hline & ST266 & $2-1-1-13-16-1-4$ & 1 & - & CTX-M-27 (1) \\
\hline & ST267 & $3-5-20-1-16-1-12$ & 2 & - & Unknown (2) \\
\hline
\end{tabular}

*Allelic profiles are in the following order: gapA, infB, $m d h, p g i, p h o E, r p o B$ and tonB.

$\dagger$ No isolates.

¥Ancestor of CC was not designated.

and bacteraemia, respectively. It was found in most hospitals except DFH; 25 isolates from SMC, nine isolates from ConNUH, four isolates from JNUH, three isolates from $\mathrm{KSH}$, three isolates from $\mathrm{CunNUH}$, two isolates from GNUH, one isolate from $\mathrm{KUH}$ and one isolate from CubNUH. Although these ST11 isolates showed similar PFGE results, they may diversify into several subtypes. Specific PFGE patterns were not related with a specific hospital or a specific ESBL gene. Only one to three isolates belonged to the other 21 STs. Thirty-two ST11 isolates contained one or more ESBL genes, consisting of eight combinations (Table 4). Although it was investigated whether other ESBL genes such as OXA and VEB were present in them, we could not find additional ESBL genes. ST11 has been described in Europe such as Hungary, the Netherlands, France and Spain (http://
www.pasteur.fr/recherche/genopole/PF8/mlst/) and designated epidemic clone III (EC III) (Damjanova et al., 2008). Dissemination in both Europe and Asia may indicate that ST11 is one of the pandemic clones although there are limited epidemiological data on the worldwide distribution of $K$. pneumoniae. These ST11 isolates from Korea are characterized by all being ciprofloxacinresistant, while only $25.0 \%$ of other ESBL-producing isolates were resistant to ciprofloxacin (data not shown). However, the combination of different ESBL genes with ST11 isolates may indicate that the ESBL-producing isolates with ST11 have diverse origins. In addition, it can be emphasized that further characterization of ESBL-producing isolates such as assay of bla genes is needed, instead of relying only on the MLST, ribotyping, PFGE, etc. 
The most prevalent ESBL in ST11 isolates was SHV-12 (13 isolates), followed by SHV-31 (11 isolates). Among 17 ESBL-producing isolates with SHV-12, 13 isolates (76.5\%) belonged to ST11. Isolates with CTX-M-15 showed divergent genotypes. Another prevalent CTX-M enzyme, CTX-M-14, was also found in diverse clones: nine isolates with CTX-M-14 belonged to six STs. CTX-M-type ESBLs, especially CTX-M-15, have recently emerged as the prevailing ESBL enzyme in Gram-negative bacteria including K. pneumoniae (Livermore et al., 2007). In the present study, CTX-M-15-producing isolates showed eight different STs. Although outbreaks of clonal strains producing CTX-M-15 have been reported worldwide, several reports also documented clonal diversity of CTXM-15 based on PFGE type (Abbassi et al., 2008; Livermore et al., 2007), which suggests horizontal transfer of the $b l a_{\text {CTX-M-15 }}$ gene. In addition, MLST analyses also indicated that CTX-M-15-producing $K$. pneumoniae isolates belonged to diverse STs (Elhani et al., 2010; Oteo et al., 2009). Moreover, different STs were positive for $b l a_{\text {CTX-M- }}$ ${ }_{15}$ in each country: STs 1, 11, 14, 17, 20, 35 and 36 in Spain (Oteo et al., 2009); and STs 42, 101, 104, 133, 147 and 321 (Elhani et al., 2010), and STs 11, 15, 17, 34, 37, 48, 218 and 271 in Korea (this study). Therefore, $b l a_{\text {CTX-M-14 }}$ or bla $a_{\mathrm{CTX}}$ $\mathrm{M}-15$ genes might be incorporated frequently into several $\mathrm{K}$. pneumoniae strains including the inter-continently disseminated clone ST11.

Although it was recently reported that most $K$. pneumoniae isolates causing liver abscess in Korea were ST23, serotype K1 and magA-positive (Chung et al., 2008), no ST23 ESBLproducing isolates were found in this study, which suggests that different $K$. pneumoniae infections might be caused by different clones.

Our study may have a few limitations: (i) sufficient $K$. pneumoniae isolates were not included although 10 hospitals participated in the surveillance; and (ii) the numbers of included isolates were not equal among participating hospitals. However, we propose that ST11 is prevalent in ESBL-producing $K$. pneumoniae isolates causing bacteraemia and UTIs in Korean hospitals, although a large study including more isolates from more hospitals is required. Isolates of the same ST such as ST11 carried different ESBL genes, which may indicate that more tools employed in the epidemiological study would guarantee better understanding of the evolution of ESBL-producing isolates and tracking of them. In addition, CTX-M-type ESBLs belonged to diverse STs, indicating their frequent introduction into K. pneumoniae.

\section{ACKNOWLEDGEMENTS}

This study was supported by a grant from the Korea Center for Disease Control and Prevention (Serial Number 2007-E00037-00) and the Asian-Pacific Research Foundation for Infectious Diseases (ARFID) (Seoul, Korea).

\section{REFERENCES}

Abbassi, M. S., Torres, C., Achour, W., Vinué, L., Sáenz, Y., Costa, D., Bouchami, O. \& Ben Hassen, A. (2008). Genetic characterization of CTX-M-15-producing Klebsiella pneumoniae and Escherichia coli strains isolated from stem cell transplant patients in Tunisia. Int $J$ Antimicrob Agents 32, 308-314.

Bae, I. K., Lee, Y. N., Jeong, S. H., Lee, K., Lee, H., Kwak, H. S. \& Woo, G. J. (2007). High prevalence of SHV-12 and the emergence of CTXM-12 in clinical isolates of Klebsiella pneumoniae from Korea. Int J Antimicrob Agents 29, 362-364.

Brisse, S., Fevre, C., Passet, V., Issenhuth-Jeanjean, S., Tournebize, R., Diancourt, L. \& Grimont, P. (2009). Virulent clones of Klebsiella pneumoniae: identification and evolutionary scenario based on genomic and phenotypic characterization. PLoS One 4, e4982.

Chung, D. R., Lee, H. R., Lee, S. S., Kim, S. W., Chang, H. H., Jung, S. I., Oh, M. D., Ko, K. S., Kang, C. I. \& other authors (2008). Evidence for clonal dissemination of the serotype K1 Klebsiella pneumoniae strain causing invasive liver abscesses in Korea. J Clin Microbiol 46, 4061-4063.

CLSI (2009). Performance Standards for Antimicrobial Susceptibility Testing, 19th Informational Supplement. M100-S19. Wayne, PA: Clinical and Laboratory Standards Institute.

Damjanova, I., Tóth, A., Pászti, J., Hajbel-Vékony, G., Jakab, M., Berta, J., Milch, H. \& Füzi, M. (2008). Expansion and countrywide dissemination of ST11, ST15, and ST147 ciprofloxacin-resistant CTXM-15-type $\beta$-lactamase-producing Klebsiella pneumoniae epidemic clones in Hungary in 2005 - the new 'MRSAs'? J Antimicrob Chemother 62, 978-985.

Diancourt, L., Passet, V., Verhoef, J., Grimont, P. A. \& Brisse, S. (2005). Multilocus sequence typing of Klebsiella pneumoniae nosocomial isolates. J Clin Microbiol 43, 4178-4182.

Elhani, D., Bakir, L., Aouni, M., Passet, V., Arlet, G., Brisse, S. \& Weill, F. X. (2010). Molecular epidemiology of extended-spectrum betalactamase-producing Klebsiella pneumoniae strains in a university hospital in Tunis, Tunisia, 1999-2005. Clin Microbiol Infect 16, 157-164.

Feil, E. J., Li, B. C., Aanensen, D. M., Hanage, W. P. \& Spratt, B. G. (2004). eBURST: inferring patterns of evolutionary descent among clusters of related bacterial genotypes from multilocus sequence typing. J Bacteriol 186, 1518-1530.

Girlich, D., Poirel, L., Leelaporn, A., Karim, A., Tribuddharat, C., Fennewald, M. \& Nordmann, P. (2001). Molecular epidemiology of the integron-located VEB-1 extended spectrum $\beta$-lactamase in nosocomial enterobacterial isolates in Bangkok, Thailand. J Clin Microbiol 39, 175-182.

Jeong, S. H., Bae, I. K., Kwon, S. B., Lee, J. H., Jung, H. I., Song, J. S., Jeong, B. C., Kim, S. J. \& Lee, S. H. (2004). Investigation of extendedspectrum $\beta$-lactamases produced by clinical isolates of Klebsiella pneumoniae and Escherichia coli in Korea. Lett Appl Microbiol 39, 41-47.

Jones, M. E., Karlowsky, J. A., Draghi, D. C., Thornberry, C., Sahm, D. F. \& Bradley, J. S. (2004). Rates of antimicrobial resistance among common bacterial pathogens causing respiratory, blood, urine, and skin and soft tissue infections in pediatric patients. Eur J Clin Microbiol Infect Dis 23, 445-455.

Kim, J., Lim, Y. M., Rheem, I., Lee, Y., Lee, J. C., Seol, S. Y., Lee, Y. C. \& Cho, D. T. (2005). CTX-M and SHV-12 $\beta$-lactamases are the most common extended-spectrum enzymes in clinical isolates of Escherichia coli and Klebsiella pneumoniae collected from 3 university hospitals within Korea. FEMS Microbiol Lett 245, 93-98.

Ko, K. S., Lee, M. Y., Song, J. H., Lee, H., Jung, D. S., Jung, S. I., Kim, S. W., Chang, H. H., Yeom, J. S. \& other authors (2008a). Prevalence and characterization of extended-spectrum $\beta$-lactamase-producing 
Enterobacteriaceae isolated in Korean hospitals. Diagn Microbiol Infect Dis 61, 453-459.

Ko, K. S., Yeom, J. S., Lee, M. Y., Peck, K. R. \& Song, J. H. (2008b). Clonal dissemination of extended-spectrum $\beta$-lactamase (ESBL)producing K. pneumoniae isolates in a Korean hospital. J Korean Med Sci 23, 53-60.

Kolar, M., Latal, T., Cermak, P., Bartonikova, N., Chmelarova, E., Sauer, P. \& Kesselova, M. (2006). Prevalence of extended-spectrum $\beta$-lactamase-positive Klebsiella pneumoniae isolates in the Czech Republic. Int J Antimicrob Agents 28, 49-53.

Lee, K., Chong, Y., Shin, H. B., Kim, Y. A., Yong, D. \& Yum, J. H. (2001). Modified-Hodge and EDTA-disk synergy tests to screen metallo-beta-lactamase-producing strains of Pseudomonase and Acinetobacter species. Clin Microbiol Infect 7, 88-91.

Livermore, D. M., Canton, R., Gniadkowski, M., Nordmann, P., Rossolini, G. M., Arlet, G., Ayala, J., Coque, T. M., Kern-Zdanowicz, I. \& other authors (2007). CTX-M: changing the face of ESBLs in Europe. J Antimicrob Chemother 59, 165-174.
Maiden, M. C., Bygraves, J. A., Feil, E., Morelli, G., Russell, J. E., Urwin, R., Zhang, Q., Zhou, J., Zurth, K. \& other authors (1998). Multilocus sequence typing: a portable approach to identification of clones within populations of pathogenic microorganisms. Proc Natl Acad Sci U S A 95, 3140-3145.

Oteo, J., Cuevas, O., López-Rodríguez, I., Banderas-Florido, A., Vindel, A., Pérez-Vázquez, M., Bautista, V., Arroyo, M., GarcíaCaballero, J. \& other authors (2009). Emergence of CTX-M-15producing Klebsiella pneumoniae of multilocus sequence types 1, 11, $14,17,20,35$ and 36 as pathogens and colonizers in newborns and adults. J Antimicrob Chemother 64, 524-528.

Poirel, L., Lartigue, M.-F., Decousser, J.-W. \& Nordmann, P. (2005). ISEcp1B-mediated transposition of bla $a_{\mathrm{CTX}-\mathrm{M}}$ in Escherichia coli. Antimicrob Agents Chemother 49, 447-450.

Ryoo, N. H., Kim, E. C., Hong, S. G., Park, Y. J., Lee, K., Bae, I. K., Song, E. H. \& Jeong, S. H. (2005). Dissemination of SHV-12 and CTX-M-type extended-spectrum $\beta$-lactamases among clinical isolates of Escherichia coli and Klebsiella pneumoniae and emergence of GES-3 in Korea. J Antimicrob Chemother 56, 698-702. 